\title{
Regulation of programmed-death ligand in the human head and neck squamous cell carcinoma microenvironment is mediated through matrix metalloproteinase-mediated proteolytic cleavage
}

\author{
MAYUKO HIRA-MIYAZAWA ${ }^{1}$, HIROYUKI NAKAMURA ${ }^{1}$, MARIKO HIRAI ${ }^{1}$, YUTAKA KOBAYASHI ${ }^{1}$, \\ HIROKO KITAHARA $^{1}$, GEORGE BOU-GHARIOS ${ }^{2}$ and SHUICHI KAWASHIRI ${ }^{1}$ \\ ${ }^{1}$ Department of Oral and Maxillofacial Surgery, Division of Cancer Medicine, \\ Kanazawa University Graduate School of Medical Science, Kanazawa, Ishikawa 920-8640, Japan; \\ ${ }^{2}$ Institute of Ageing and Chronic Disease, University of Liverpool, Liverpool L7 8TX, UK
}

Received September 26, 2017; Accepted December 7, 2017

DOI: 10.3892/ijo.2017.4221

\begin{abstract}
Recurrent and/or metastatic head and neck squamous cell carcinoma (R/M HNSCC) is a devastating malignancy with a poor prognosis. According to recent clinical studies, tumour growth can be effectively reduced and survival can be improved by blocking the programmed death receptor-1 (PD-1)/programmed death-ligand 1 (PD-L1) pathway. PD-L1 expression has been proposed as a potential causative mechanism, as HNSCC is highly immunosuppressive. However, anti-PD-1 treatment is beneficial only for certain patients. Therefore, the mechanisms controlling PD-L1 expression warrant further investigation in order to provide a better understanding of the predicting efficacy of and optimising anti-PD-1 therapy, alone or in combination. In this study, PD-L1 protein extracted from the cell membrane was found to be downregulated in OSC-20 cells compared with OSC-19 cells, despite a higher PD-L1 expression in the total cell lysate of the OSC-20 compared with the OSC-19 cells. Several matrix metalloproteinases (MMPs) were found to be upregulated in HNSCC; in particular, MMP-7 and -13 were upregulated in
\end{abstract}

Correspondence to: Dr Hiroyuki Nakamura, Department of Oral and Maxillofacial Surgery, Division of Cancer Medicine, Kanazawa University Graduate School of Medical Science, 13-1 Takara-machi, Kanazawa, Ishikawa 920-8640, Japan

E-mail: hnak@me.com

Abbreviations: DCs, dendritic cells; HAL, eribulin mesylate (also known as Halaven); HNSCC, head and neck squamous cell carcinoma; PTX, paclitaxel; MMP, matrix metalloproteinase; PD-1, programmed death receptor-1; PD-L1, programmed death-ligand 1; PBMCs, peripheral blood mononuclear cells; qPCR, quantitative (real-time) polymerase chain reaction; R/M HNSCC, recurrent and/or metastatic head and neck squamous cell carcinoma; VBL, vinblastine

Key words: recurrent and/or metastatic head and neck squamous cell carcinoma, programmed death receptor 1, programmed death-ligand-1, matrix metalloproteinase, tumour microenvironment the OSC-20 compared with the OSC-19 cells. Purified PD-L1 was degraded by recombinant MMP-13 and -7. The expression of PD-L1 was significantly restored by a specific inhibitor of MMP-13 (CL82198), which suggested the involvement of MMP-13 in the shedding/cleavage of PD-L1 in the OSC-20 cells. Among the anticancer drugs conventionally used in the treatment of patients with HNSCC, paclitaxel increased MMP-13 expression in R/M HNSCC cells (HOC313 cells) co-cultured without/with dendritic cells (DCs). These results suggest that the shedding/cleavage of PD-L1 by MMP-13 is one of the mechanisms behind the protective effect against invasion and metastasis. Thus, MMP-13 has potential value as a marker predictive of the decreased efficacy of anti-PD-1 therapy. In addition, paclitaxel is a particularly promising candidate for combination therapy in R/M HNSCC with antiPD-1 therapy.

\section{Introduction}

Recurrent and/or metastatic head and neck squamous cell carcinoma (R/M HNSCC) is a devastating malignancy with a poor prognosis. Treatment is limited to chemotherapeutic approaches. In recent studies, the programmed-death receptor-1 (PD-1 and CD279)/programmed-death ligand 1 (PD-L1, B7-H1 and CD274) pathway has been indicated to be critical for regulating T-cell responses and maintaining immune suppression (1-4). HNSCC is a malignant tumour found most often in the head and neck region involving the mandibular gingiva and oral floor $(5,6)$; it is generally highly immunosuppressive, for which PD-L1 expression has been proposed as a potential causative mechanism (7-9). In many tumours, expression of PD-L1 is constitutive and is induced by interferon- $\gamma($ IFN- $\gamma)$. PD-L1 binds to an inhibitory receptor (PD-1), which is a member of the B7 family of receptors (4), and to the co-stimulatory molecule, CD80 (B7-1) (10). PD-1 is expressed on activated T cells; when PD-1 ligates with tumour-associated PD-L1, the apoptosis or downregulation of effector cytotoxic $\mathrm{T}$ lymphocytes is induced, thereby resulting in an escape from T-cell-mediated immune surveillance (11). Tumour growth is reduced and survival is improved when the 
PD-L1/PD-1 pathway is blocked (7,12-15). Moreover, clinical studies have indicated that tumour regression by the blockade of the PD-1/PD-L1 checkpoint is durable $(1,2)$, leading to the recent registration of an anti-PD-1 antibody for head and neck cancer. In treated tumours, the response to the PD-1 blockade has been commonly been associated with PD-L1 expression (1).

However, anti-PD-1 treatment appears to be beneficial only in certain patients. Therefore, the mechanisms underlying the unknown regulation of PD-L1 expression in the HNSCC microenvironment remain to be elucidated. PD-L1 expression has been shown to be lower in R/M HNSCC cell lines than in a low-grade invasive and less metastatic HNSCC cell lines, and PD-L1 expression and epithelial-mesenchymal transition (EMT) have been found to be closely related (16). PD-L1 expression has been shown to be upregulated in macrophages and dendritic cells (DCs) in human R/M HNSCC tissues or co-cultured with R/M HNSCC cells in vitro (16). However, the mechanisms responsible for the fact that PD-L1-expressing HNSCC cells exhibit low invasiveness and are less metastatic remain to be determined.

The immunosuppressive capacity of PD-1 ligands on fibroblasts may be limited by their matrix metalloproteinase (MMP)-dependent cleavage, thereby contributing to the aggravation of inflammation in tissues (17). Conversely, MMP activity seems to deplete PD-1 ligands in carcinomaassociated fibroblasts, which may impair the physical deletion of exhausted defective memory $\mathrm{T}$ cells through apoptosis and may facilitate their regulatory functions (17). As MMPs are a group of proteolytic enzymes that can degrade principal components of the extracellular matrix, they are widely believed to play an important role in tissue degradation. Several sets of experimental and clinical data concerning MMPs in the contexts of cancer have been reported $(18,19)$. Numerous MMP inhibitors have exhibited efficacy in animal models of disease and have been used in clinical trials in the treatment of cancer, with some studies focusing on rheumatoid arthritis and osteoarthritis. However, MMP inhibitors have not exhibited significant therapeutic effects in any of these human clinical trials (20). The use of these inhibitors also results in adverse effects, including musculoskeletal pain, tendonitis and mild anaemia with elevated liver enzyme levels (20). Therefore, the function of MMP needs to be redefined. MMPs influence basic processes, such as cell proliferation, differentiation, angiogenesis and apoptosis (18). Notably, the MMP family of proteins exert dual roles in the pathogenesis of inflammation: Stimulating protective innate and/or adaptive immune functions, as well as tissue destruction (21).

To predict the efficacy of and optimise anti-PD-1 therapy, alone or in combination with other treatment options, it is important to elucidate the mechanisms controlling PD-L1 expression. In this study, we thus focused on the regulation of PD-L1 expression in HNSCC, and discussed the mechanism of this regulation of $\mathrm{PD}-\mathrm{L} 1$ expression in the tumour microenvironment.

\section{Materials and methods}

Cell culture. Three HNSCC cell lines originally established from tumour biopsies with different grades of invasive or metastatic abilities were used, including OSC-20 cells (with low invasiveness), OSC-19 cells (intermediate invasiveness) and HOC313 cells (recurrent high-grade invasiveness and metastasis). The OSC-20 cell line was originally derived from a 58-year-old female with tongue cancer (22). OSC-19 was derived from a 61-year-old male with tongue cancer metastatic to the cervical lymph nodes (23). HOC313 was derived from a 51-year-old female with HNSCC (involving the mandibular gingiva and oral floor) that metastasised to the cervical lymph nodes and recurred (24). The HOC313 cells were a kind gift from Dr M. Nagayama (Tokushima University, Tokushima, Japan). The OSC-20 (JCRB \#0197) and OSC-19 (JCRB \#0198) cells, and normal human oral fibroblasts of the lip mucosa (KD; JCRB \#9103) were obtained from the JCRB Cell Bank (Osaka, Japan). DCs were generated from human peripheral blood mononuclear cells (PBMCs), as previously described $(25,26)$. Experiments using human samples were approved by the Ethics Committee of the Kanazawa University Graduate School of Medical Science (IRB no. 352-2), and written informed consent was obtained from persons providing human samples. Peripheral blood was voluntarily donated by 3 healthy individuals. PBMCs were obtained by venepuncture into an 8-ml Vacutainer CPT Cell-Preparation Tube (BD Vacutainer Systems, Franklin Lakes, NJ, USA). Monocyte-derived DCs were generated by incubating monocytes at $1 \times 10^{6}$ cells/ml in G4 medium (G4 Dendritic Cell Generation kit; HumanZyme, Chicago, IL, USA) at $37^{\circ} \mathrm{C}$ in a $\mathrm{CO}_{2}(5 \%)$ incubator for 7 days. The induced DCs were examined using an anti-DC antibody (CD83; Abcam, Tokyo, Japan). Eribulin (also known as Halaven; HAL) was purchased from Eisai Co., Ltd. (Tsukuba, Japan). Vinblastine (VBL) and paclitaxel (PTX; Taxol) were purchased from Nihon Kayaku (Tokyo, Japan).

RNA extraction, cDNA synthesis and quantitative (real-time) PCR ( $q$ PCR). The mRNA expression levels of PD-L1, and MMP-1, -2, -3, -7, -8, -9, -10, -11, -12, -13 and -14 were analysed using a Rotor-Gene Q 2plex System (Qiagen, Hilden, Germany) with FAM/ZEN/IBFQ probes (Integrated DNA Technologies, Inc., Coralville, IA, USA; DNA sequences not available). Total RNA was extracted using the PureLink RNA mini kit (Thermo Fisher Scientific, Waltham, MA, USA), and cDNA was obtained using the PrimeScript First-Strand cDNA synthesis kit (Takara, Tokyo, Japan). All reactions were performed in accordance with the manufacturer's instructions. We amplified 18S rRNA as an internal standard using HEX/ZEN/IBFQ probes (Integrated DNA Technologies, Inc.; DNA sequences not available). Relative expression levels were calculated using the $\Delta \Delta C t$ method for qPCR (27), which presents the data as fold differences in expression level relative to a calibrator sample, in this case represented by the mean expression of 3 experimental measurements of 18S rRNA in the control cells or vehicle (buffer only control)-treated cells.

Western blot analysis. The concentration of total extracted protein was measured after 100 -fold $\mathrm{dH}_{2} \mathrm{O}$ dilution by the Qubit Protein assay, in accordance with the manufacturer's instructions (Thermo Fisher Scientific). An equal amount (30 $\mu \mathrm{g}$ of protein) of lysate or culture medium was mixed with loading buffer, with this mixture then being electrophoretically 
separated and transferred onto membranes. The membranes were blocked with Blocking One (Nacalai Tesque, Kyoto, Japan), followed by incubation with anti-PD-L1 or MMP-7, -12, -13 antibodies (PD-L1, cat. no. 210931; MMP-7, cat. no. 205525; MMP-12, cat. no. 52897; MMP-13, cat. no. 51072; Abcam) and an anti-human $\beta$-actin antibody (cat. no. 4970; Cell Signaling Technology, Tokyo, Japan). After washing with Tris-buffered saline (TBS) with $0.05 \%$ Tween, the membranes were incubated with horseradish peroxidase-conjugated anti-mouse IgG. After washing with TBS-0.05\% Tween-20, membranes were incubated with the ECL Prime Western Blotting Detection Reagent (GE Healthcare, Little Chalfont, UK). Signals were detected and analysed using C-DiGit (M\&S TechnoSystems, Tokyo, Japan).

Enzyme-linked immunosorbent assay (ELISA). The membrane-associated proteins were extracted separately from the cytosolic proteins using the Mem-PER Plus Membrane Protein Extraction kit (Thermo Fisher Scientific). The extracted membrane-associated proteins were analysed for the presence of PD-L1 using the ELISA kit (cat. no. DY156; R\&D Systems Europe Ltd., Abingdon, UK), in accordance with the manufacturer's instructions. The total membrane-associated protein concentration of the sample was measured by the Qubit Protein assay in accordance with the manufacturer's instructions (Thermo Fisher Scientific). Values were calculated as $\mathrm{pg} / \mathrm{mg}$ total protein or $\mathrm{ng} / \mathrm{mg}$ total protein. Data are presented as the means \pm standard error of the mean (SEM).

Expression and purification of recombinant human PD-L1. Human PD-L1 cDNA (cat. no. RDC1087) was purchased from R\&D Systems Europe Ltd. The human PD-L1 coding sequence and 6xHis-Tag were fused using PCR and the InFusion HD kit (Takara Bio) combined with pSG5 (Agilent Technologies Japan, Tokyo, Japan) to construct the expression plasmid pSG-hPDL1-His. The sequence was confirmed by sequencing.

The Expi293 cells were cultured in Expi293 Expression Medium (both from Thermo Fisher Scientific) and transfected with the pSG-hPDL1-His using FuGENE 6 transfection reagent (Promega, Tokyo, Japan). The culture media were harvested at 6 days after transfection and used for purification. They were concentrated using an Amicon Diaflo apparatus fitted with a YM-10 membrane (both from Merck Japan, Tokyo, Japan) and applied to complete His-Tag Purification Resin (Roche Diagnostics, Basel, Switzerland) equilibrated with $50 \mathrm{mM}$ Tris- $\mathrm{HCl}$ (pH 7.5), $0.15 \mathrm{M} \mathrm{NaCl}$ and $0.05 \%$ Brij 35 . Recombinant human PD-L1 was eluted with $500 \mathrm{mM}$ imidazole in $50 \mathrm{mM}$ Tris- $\mathrm{HCl}(\mathrm{pH} 7.5)$ buffer after washing the column with $50 \mathrm{mM}$ Tris- $\mathrm{HCl}(\mathrm{pH} 7.5)$ buffer containing $5 \mathrm{mM}$ imidazole and $0.3 \mathrm{M} \mathrm{NaCl}$. The combined fractions of human PD-L1 were dialysed against $50 \mathrm{mM}$ Tris- $\mathrm{HCl}(\mathrm{pH} 7.5), 0.15 \mathrm{M}$ $\mathrm{NaCl}$ and $0.05 \%$ Brij 35 buffer to remove imidazole. The protein concentration was determined using the Qubit Protein assay, in accordance with the manufacturer's instructions (Thermo Fisher Scientific). Eluted fractions containing a major species of Mr 56,000 and minor protein bands of Mr 46,000 were used as the source of PD-L1. Both minor species were recognised by an anti-PD-L1 antibody (PD-L1, cat. no. 210931; Abcam), while only major species were recognised by an antiHis antibody (cat. no. D291-3; MBL, Tokyo, Japan).
Degradation of PD-L1 by MMP-13 and -7. The digestion of PD-L1 was examined by incubation of the purified PD-L1 at $37^{\circ} \mathrm{C}$ for $24 \mathrm{~h}$ with MMP-13 or -7 at an enzyme/substrate molar ratio of 1:30 in $50 \mathrm{mM}$ Tris- $\mathrm{HCl}(\mathrm{pH} 7.5), 0.15 \mathrm{M} \mathrm{NaCl}, 10 \mathrm{mM}$ $\mathrm{CaCl}_{2}$ and $0.05 \%$ Brij 35 (TNCB buffer). Human recombinant MMP-13 (cat. no. 444287) and MMP-7 (cat. no. CC1057) were purchased from Merck Japan. MMPs were activated by incubation with p-aminophenylmercuric acetate (Merck Japan), in accordance with previously reported methods $(28,29)$. These reactions were terminated with $20 \mathrm{mM}$ ethylenediaminetetraacetic acid (EDTA) and the degradation products were examined by western blot analysis using anti-PD-L1 antibody (PD-L1, cat. no. 210931; Abcam).

Inhibition of PD-L1 cell surface degradation in HNSCC cells. For the incubation of HNSCC cells with MMP inhibitors, the HNSCC cells were seeded at a concentration of $5 \times 10^{4}$ cells $/ \mathrm{cm}^{2}$ in P6 plates in DMEM medium (Sigma-Aldrich Japan, Tokyo, Japan) supplemented with 5\% FCS and were then incubated on day 1 in the presence or absence of 2 MMP inhibitors $(10 \mu \mathrm{g} / \mathrm{ml}$ CL82198, cat. no. 141576; and $10 \mu \mathrm{M}$ BB94, cat. no. 142087; Abcam) and maintained in culture for 4 days at $37^{\circ} \mathrm{C}$ without a medium change. Subsequently, membrane-associated proteins were extracted separately using the Mem-PER Plus Membrane Protein Extraction kit (Thermo Fisher Scientific). The extracted membrane-associated proteins were analysed for the presence of PD-L1 using the ELISA kit (cat. no. DY156; R\&D Systems Europe Ltd.), in accordance with the manufacturer's instructions. The total protein concentration of the sample was measured after 100-fold $\mathrm{dH}_{2} \mathrm{O}$ dilution using the Qubit Protein assay, in accordance with the manufacturer's instructions (Thermo Fisher Scientific). Values were calculated as pg/mg total protein or $\mathrm{ng} / \mathrm{mg}$ total protein. The PD-L1 expression recovery $(\%)$ was expressed as follows: Ratio $(\%)=$ difference between the amount of PD-L1 in HNSCC cells incubated in the presence of MMP inhibitors on day 4 and the amount of PD-L1 in HNSCC cells in the absence of MMP inhibitors on day 4/the difference between the amount of PD-L1 in HNSCC cells in the absence of MMP inhibitors on day 1 and the amount of PD-L1 in HNSCC cells in the absence of MMP inhibitors on day $4 \times 100$.

Statistical analysis. For comparisons between samples, data were analysed by ANOVA and Tukey's multiple comparison tests using SPSS, version 23 software (IBM SPSS Statistics, IBM Corp., NY, USA). A P-value $\leq 0.05$ or less was accepted as statistically significant.

\section{Results}

PD-L1 expression in HNSCC cell lines and cell surface PD-L1 degradation in OSC-20 cell lines. As shown in Fig. 1A, qPCR revealed that $\mathrm{PD}-\mathrm{L} 1 \mathrm{mRNA}$ expression was upregulated in the OSC-20 and OSC-19 cells compared with that in the KD cells. Among these 3 HNSCC cell lines, the PD-L1 mRNA expression level ranged from 1.7- to 4.5-fold higher than that in KD cells (Fig. 1A). PD-L1 protein extracted from the total cell lysate was also upregulated in the OSC-20 and OSC-19 cells compared with the level in the HOC 313 and KD cells, as determined by western blot anaysis (Fig. 1B). However, the amount 
of PD-L1 protein extracted from the OSC-20 cell membrane (22.3-fold higher than that in the KD cells) was less than that of PD-L1 protein from the OSC-19 cell membrane (96.08-fold higher than that in KD cells) (Fig. 1C). PD-L1 protein in the culture supernatant from the OSC-20, OSC-19, HOC313 and KD cells was not detected by ELISA (data not shown).

MMP expression in HNSCC cell lines (OSC-20, OSC-19 and HOC313). As shown in Fig. 2A, qPCR was used to determine MMP-1, $-2,-3,-7,-8,-9,-10,-11,-12,-13$ and -14 mRNA expression in the OSC-20, OSC-19, HOC 313 and KD cells. The expression levels of MMP-7, $-10,-12$ and -13 in the OSC-20 cells were 2801.5-, 41.57-, 513.7- and 2522.2-fold higher than those in the KD cells, respectively. Moreover, the expression levels of MMP-9, -10 and -13 in the OSC-19 cells were 317.6-, 38.6- and 1380.7-fold higher than those in the KD cells, respectively. MMP-3 expression in the HOC313 cells was also 7.82-fold higher than that in KD cells. Overall, MMP expression in the HOC 313 cells was not markedly high compared with the levels in the OSC-20 and OSC-19 cells. Among these MMPs that were upregulated in these HNSCC cells, the expression of MMP-7, -12 and -13 in particular was markedly upregulated in the OSC-20 cells compared with that in the OSC-19 cells. As shown in Fig. 2B, western blot analysis revealed that MMP-7 and -13 protein expression levels in the culture supernatant were upregulated in the OSC-20 cells compared with those in the OSC-19 cells. MMP-12 protein expression was negligible (Fig. 2B).

Purification and digestion of PD-L1 by recombinant MMP-7 and MMP-13. PD-L1 purified from the culture medium of Expi293 cells transfected with the human PD-L1 expression vector contained a major protein band of $\mathrm{Mr} 56,000$ and a minor band of $\mathrm{Mr}$ 46,000 (Fig. 3A), both of which were recognised by anti-PD-L1 antibody. However, only the major species of $\mathrm{Mr} 56,000$ was recognised by anti-His antibody. These findings suggested that the species of $\mathrm{Mr} 46,000$ was generated by C-terminal truncation of the species of $\mathrm{Mr} 56,000$.

As shown in Fig. 2, MMP-7 and MMP-13 expression was upregulated in the OSC-20 cells, in which PD-L1 cell surface degradation was enhanced, and MMP-12 protein expression was negligible (Fig. 2B). Although MMP-12 mRNA was upregulated in the OSC-20 cells, the absolute amount of mRNA may be low. These results suggested that MMP-7 and MMP-13 were the candidates of proteinases involved in PD-L1 degradation. Recombinant MMP-7 and MMP-13 were activated by incubation with p-aminophenylmercuric acetate, in accordance with previously described methods $(28,29)$. PD-L1 was digested for $24 \mathrm{~h}$ with MMP-7 or -13, and the digestion fragments were examined by western blot analysis. MMP-7 and -13 degraded PD-L1 successfully as the amounts of PD-L1 species of Mr 56,000 and 46,000 were reduced (Fig. 3B). The digested fragments of PD-L1 were not recognised by the antiPD-L1 antibody.

Inhibition of PD-L1 cell surface degradation in HNSCC cells. To determine which MMP contributes to PD-L1 degradation in HNSCC cells, we used MMP inhibitors with different specificities. As shown in Fig. 4, only the MMP-13-specific inhibitor (CL82198) significantly $(\mathrm{P}<0.05)$ restored PD-L1 expression in
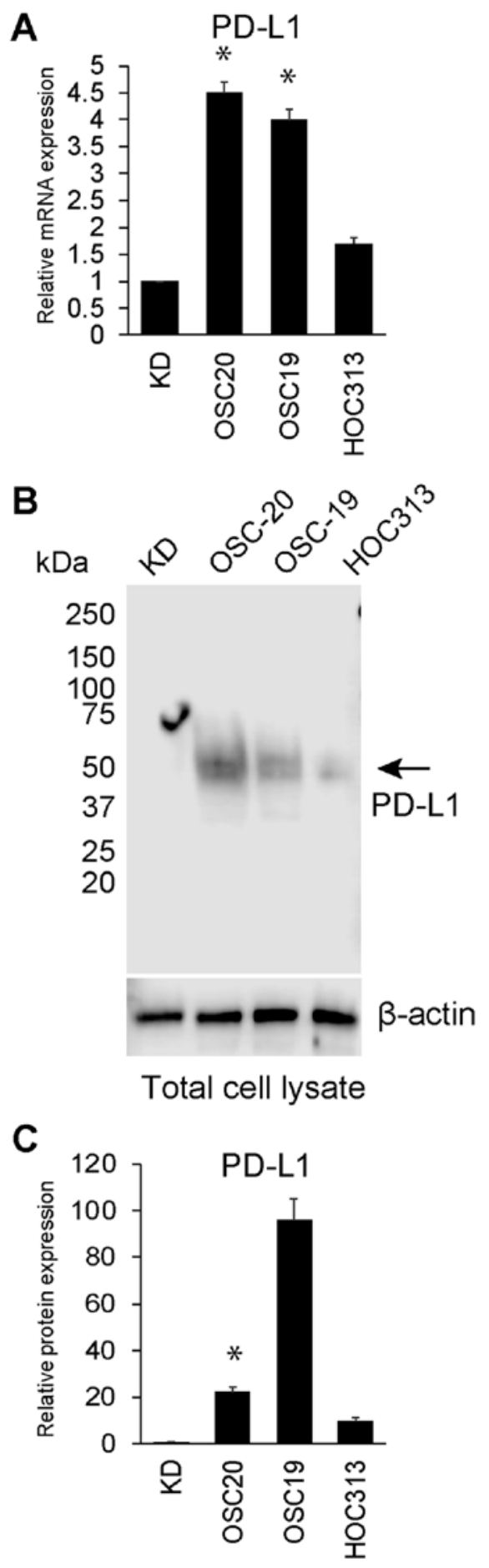

Cell membrane lysate

Figure 1. Expression and cell surface degradation of programmed death-ligand 1 (PD-L1) in head and neck squamous cell carcinoma (HNSCC) cell lines. (A) Relative mRNA expression levels of PD-L1 in the OSC-20, OSC-19, HOC313 and KD cell lines. Expression levels are displayed as fold differences relative to control cells (KD cells). Each data point represents the mean of 3 independent experiments. The vertical bars show standard deviations. Error bars correspond to SEM. ${ }^{*} \mathrm{P}<0.05$ vs. control KD cells. (B) PD-L1 protein extracted from the total cell lysate of OSC-20, OSC-19, HOC313 and KD cells was assessed by western blot analysis. The arrow indicates PD-L1. $\beta$-actin was detected as a loading control. (C) The amount of PD-L1 protein extracted from the cell membrane lysate of OSC-20, OSC-19, HOC313 and KD cells was measured by enzyme-linked immunosorbent assay (ELISA). Expression levels are displayed as fold differences relative to control cells (KD cells). Each data point represents the mean of 3 independent experiments. The vertical bars show standard deviations. Error bars correspond to SEM. ${ }^{*} \mathrm{P}<0.05$ vs. OSC- 19 cells. 
A
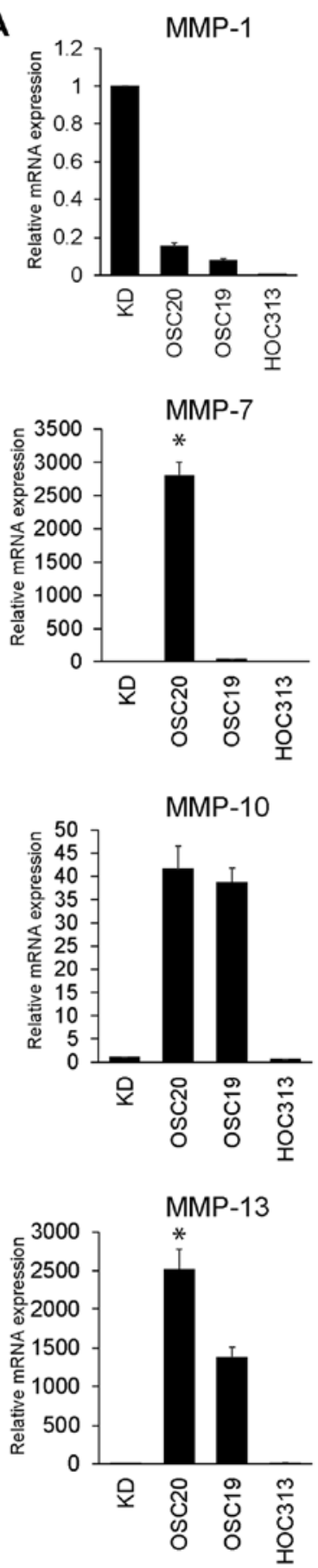

MMP-2

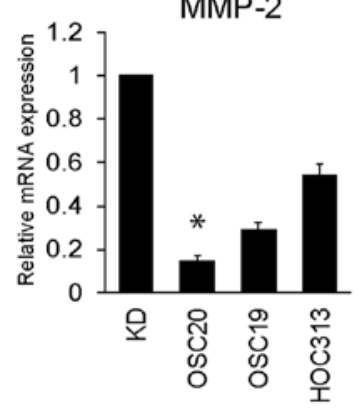

MMP-8

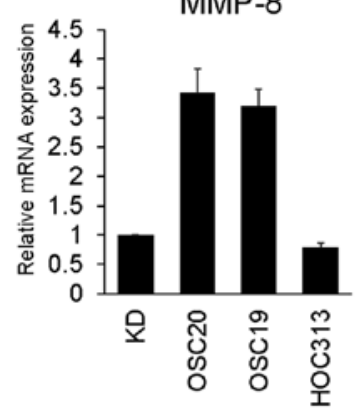

MMP-11

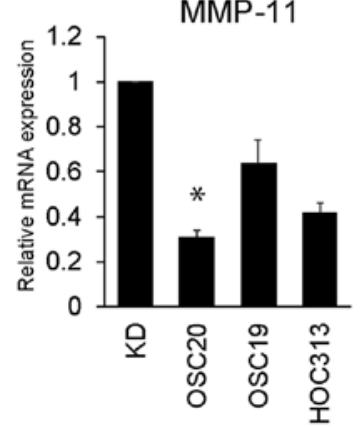

MMP-14

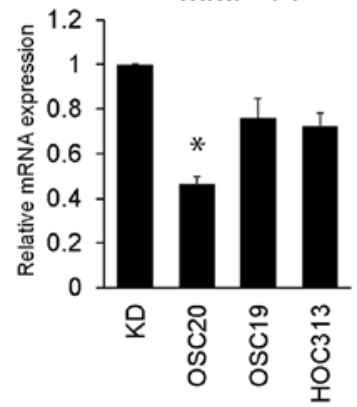

MMP-3

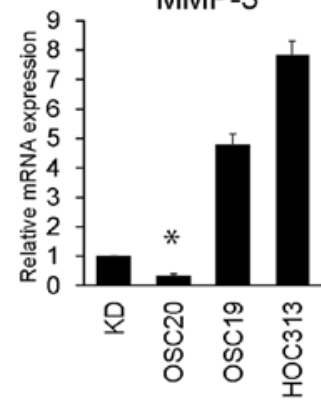

MMP-9
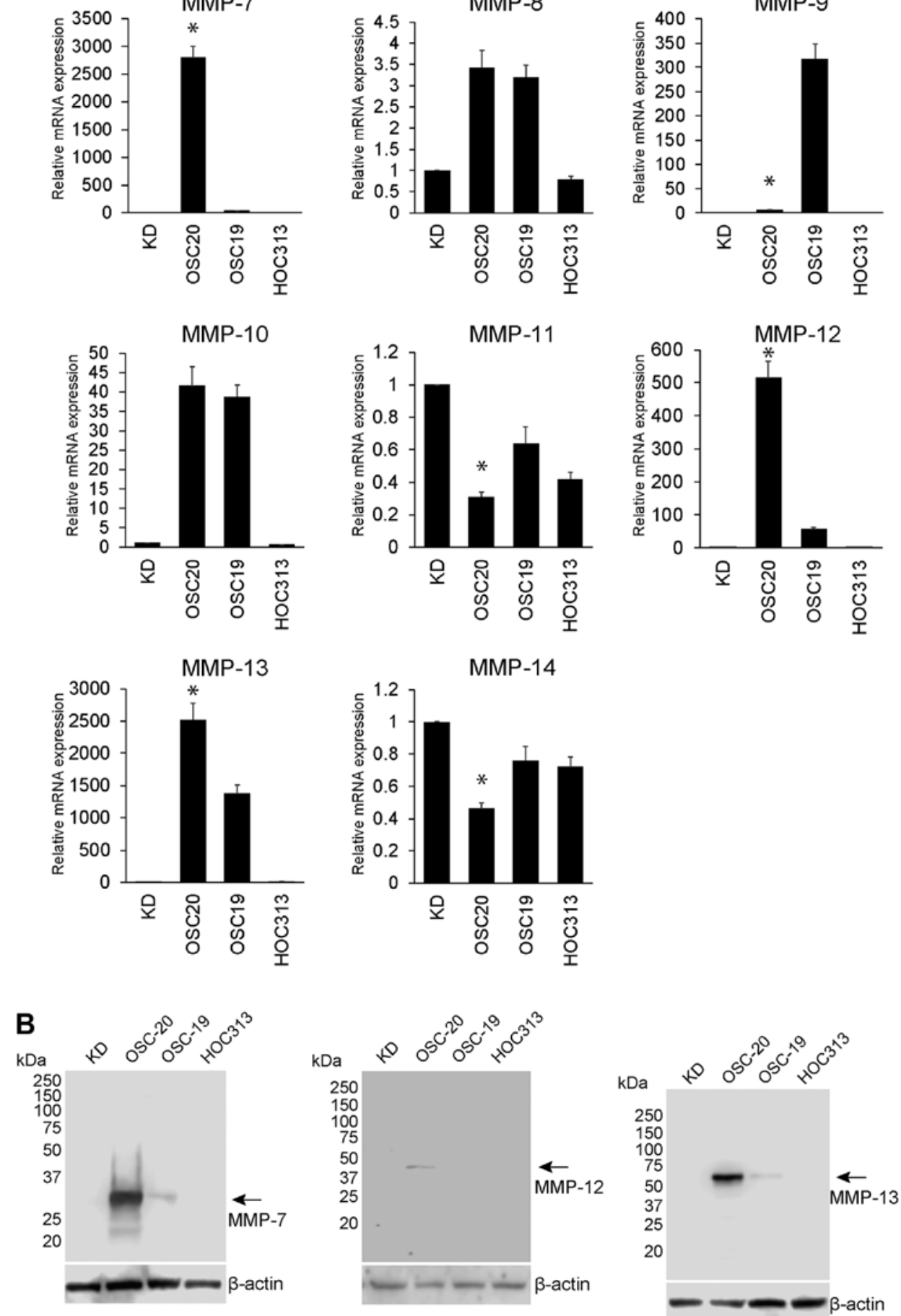

Figure 2. Matrix metalloproteinase (MMP) expression in head and neck squamous cell carcinoma (HNSCC) cell lines (OSC-20, OSC-19 and HOC313). (A) Relative mRNA expression levels of MMP-1, -2, -3, -7, -8, -9, -10,-11,-12,-13 and -14 in the OSC-20, OSC-19, HOC313 and KD cell lines. Expression levels are displayed as fold differences relative to control cells (KD cells). Each data point represents the mean of 3 independent experiments. The vertical bars show standard deviations. Error bars correspond to SEM. ${ }^{*} \mathrm{P}<0.05$ vs. OSC-19 cells. (B) MMP-7, -12 and -13 proteins in the culture supernatant of OSC-20, OSC-19, HOC313 and KD cells were assessed by western blot analysis. The arrows indicate MMP-7, -12 and -13. $\beta$-actin was detected as a loading control. 

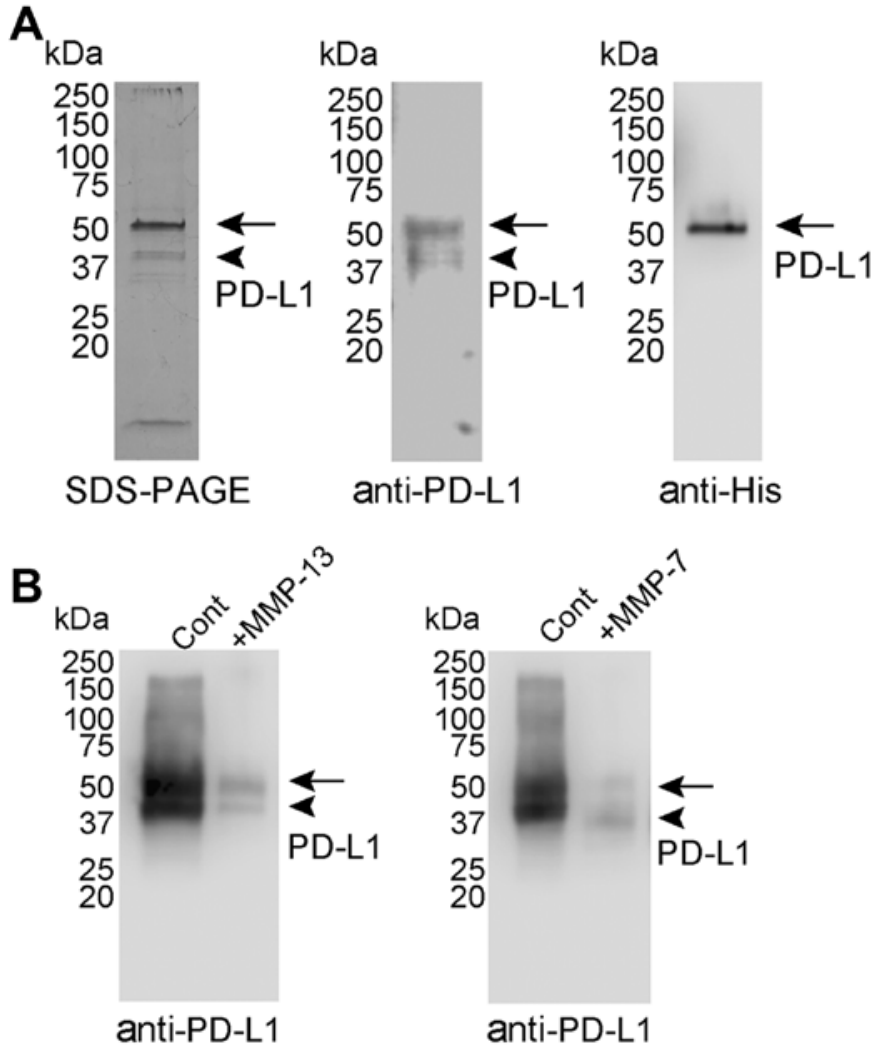

anti-PD-L1

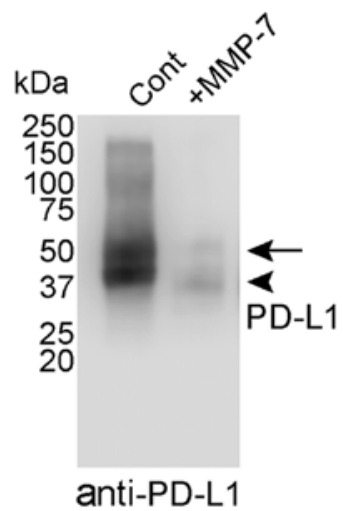

Figure 3. Purification and digestion of programmed death-ligand 1 (PD-L1) by recombinant matrix metalloproteinase-7 (MMP-7) and MMP-13. (A) Purified PD-L1 was analysed by sodium dodecyl sulfate-polyacrylamide gel electrophoresis (SDS-PAGE) (silver staining, left panel) and western blot analysis (middle and right panels) using anti-PD-L1 or anti-His antibodies, respectively. Arrows indicate PD-L1 of Mr 56,000, and arrowheads indicate PD-L1 fragment of Mr 46,000. (B) PD-L1 was incubated with MMP-13 or 7 for $24 \mathrm{~h}$ at $37^{\circ} \mathrm{C}$. The reaction products were subjected to western blot analysis using anti-PD-L1 antibody after termination of the reaction with $20 \mathrm{mM}$ ethylenediaminetetraacetic acid (EDTA). Arrows indicate PD-L1 and arrowheads indicate the PD-L1 fragment.

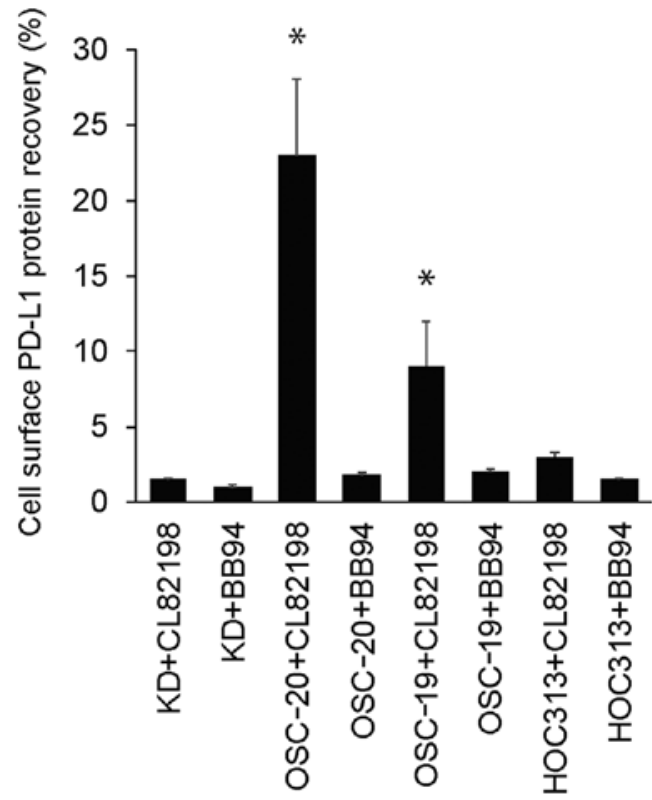

Figure 4. Inhibition of programmed death-ligand 1 (PD-L1) cell surface degradation in head and neck squamous cell carcinoma (HNSCC) cells. Recovery (\%) of PD-L1 on HNSCC cells after 4 days of culture in the presence of matrix metalloproteinase (MMP) inhibitors. After 1 day of culture at $37^{\circ} \mathrm{C}$, HNSCC cells were further cultured for 4 days at $37^{\circ} \mathrm{C}$ in the presence or absence of MMP inhibitors (without any medium change). Cell surface PD-L1 was extracted and measured by enzyme-linked immunosorbent assay (ELISA) on days 1 and 4. Each data point represents the mean of 3 independent experiments. The vertical bars show standard deviations. Error bars correspond to SEM. ${ }^{*} \mathrm{P}<0.05$ vs. BB94-treated OSC-20 and OSC-19 cells, respectively. the OSC-20 cells $(23.2 \pm 5.1 \%$ recovery from day 1 to 4 in the presence of $10 \mathrm{mg} / \mathrm{ml}$ CL82198) when compared with the BB94 inhibitor. PD-L1 expression was also significantly $(\mathrm{P}<0.05)$ restored by CL 82198 in the OSC-19 cells $(9.1 \pm 3.2 \%$ recovery from day 1 to 4 in the presence of $10 \mathrm{mg} / \mathrm{ml}$ CL82198). BB94 did not restore PD-L1 expression in the HNSCC cells.

Tubulin inhibitor increased MMP-13 expression in $R / M$ HNSCC cells (HOC313 cells) co-cultured with DCs. The results shown in Fig. 4 suggest that MMP-13 plays a key role in the shedding/cleavage of PD-L1. We wished to deterimine which anti-head and neck cancer drug would successfully upregulated MMP-13 expression. For this purpose, we used tubulin inhibitors (HAL, PTX and VBL) that are generally used in the treatment of head and neck cancer. As shown in Fig. 5A-C, following treatment of the OSC-20, OSC-19 and HOC 313 cells with tubulin inhibitors, the PD-L1 and MMP13 expression levels were analysed. MMP-13 expression was significantly downregulated in the HAL-treated OSC-20 and OSC-19 cells. Moreover, the MMP-13 and PD-L1 expression levels were significantly downregulated in the VBL-treated OSC-19 cells. No tubulin inhibitor used in the OSC-20 and OSC-19 cells increased MMP-13 expression (Fig. 5A and B). Only treatment with PTX upregulated MMP-13 expression in the HOC313 cells when compared with the other tubulin inhibitors used (Fig. 5C). No tubulin inhibitor used to treat the HOC 313 cells altered the expression level of PD-L1. As shown in Fig. 5D, we examined the effects of tubulin inhibitor 
A
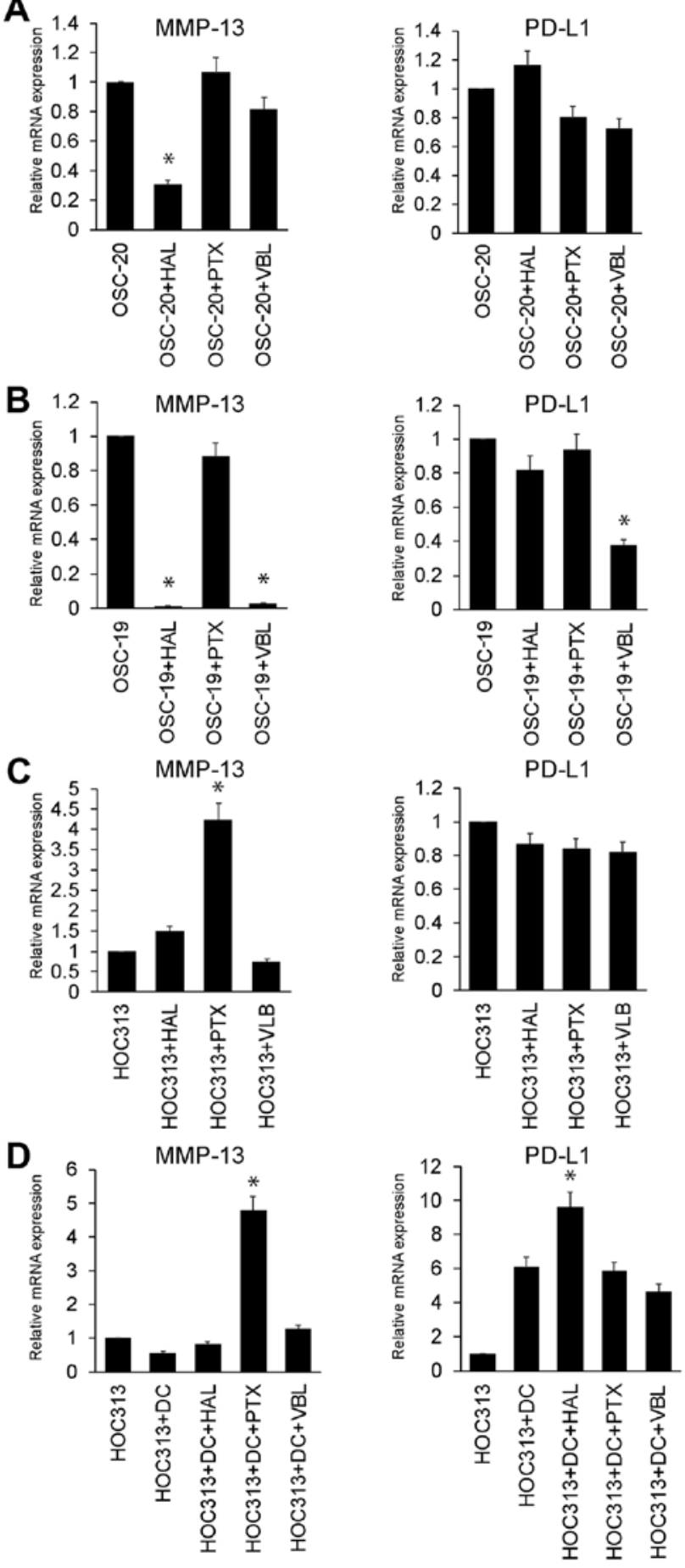

Figure 5. Tubulin inhibitor increases matrix metalloproteinase-13 (MMP-13) expression on R/M head and neck squamous cell carcinoma (HNSCC) cells (HOC313 cells) co-cultured without/with dendritic cells (DCs). Relative mRNA expression levels of MMP-13 and programmed death-ligand 1 (PD-L1) in the (A) OSC-20, (B) OSC-19 and (C) HOC313 cell lines treated with tubulin inhibitor (HAL, eribulin; PTX, paclitaxel; and VBL, vinblastine). Expression levels are displayed as fold differences relative to the control cells [vehicle (control buffer only)-treated OSC-20, OSC-19 and HOC313 cells, respectively]. Each data point represents the mean of 3 independent experiments. The vertical bars show standard deviations. Error bars correspond to SEM. ${ }^{*} \mathrm{P}<0.05$ vs. control vehicle-treated OSC-20, OSC-19 and HOC313 cells, respectively. (D) Relative mRNA expression levels of MMP-13 and PD-L1 in the DCs co-cultured with HOC313 cell lines treated with tubulin inhibitor (HAL, eribulin; PTX, paclitaxel; and VBL, vinblastine). Expression levels are displayed as fold differences relative to the control cells (control vehicle-treated HOC 313 cells). Each data point represents the mean of 3 independent experiments. The vertical bars show standard deviations. Error bars correspond to SEM. * $\mathrm{P}<0.05$ vs. control vehicle-treated DCs co-cultured with HOC 313 cells. on DCs co-cultured with HOC313 cells. Under co-culture conditions using DCs and HOC313 cells, treatment with PTX also upregulated MMP-13 expression when compared with the other tubulin inhibitors used. Treatment with HAL significantly upregulated PD-L1 expression in the HOC313 cells co-cultured with DCs.

\section{Discussion}

PD-L1 is a ligand of the inhibitory receptor, PD-1, and the PD-1/PD-L1 pathway induces and maintains peripheral immunotolerance in cancer tissues $(11,30)$. PD-L1 expression is constitutive in various types of human cancer, including squamous cell carcinomas of the lung, oesophagus, and head and neck, as well as other types of carcinomas of the colon, ovaries, bladder and breast, and melanoma and glioma (7,12,13,30-38). Our previous study demonstrated that PD-L1 expression in HNSCC cell lines with low invasiveness (OSC-20 and OSC-19 cells) was higher than that in the R/M HNSCC line (TSU cells) (16). These findings contradicted previous results for other tumour types $(7,12,13,30-38)$. Although the molecular mechanisms underlying this difference remain unclear, we hypothesise that they are confined to cancer subtypes with a unique molecular profile. In this study, we demonstrated that PD-L1 mRNA and total protein expression was downregulated in R/M HNSCC (HOC313 cells) and upregulated in cells with low invasiveness (OSC-20 and OSC-19). These findings are consistent with our previous results (16). Of note, in this study, PD-L1 protein extracted from the cell membrane was downregulated in OSC-20 cells compared with OSC-19 cells, despites higher PD-L1 expression in the total cell lysate of OSC20 cells compared with that in OSC-19 cells. These findings suggested that PD-L1 could be degraded on the OSC-20 cell surface by some proteinases.

A recent study from another group demonstrated that PD-L1 was cleaved by recombinant MMP-13 and MMP-9, which may limit the immunosuppressive capacity and may thus contribute to the exacerbation of inflammation in tissues (17). MMP family proteins are the most plausible PD-L1 degradation proteinases as they play dual roles in the pathogenesis of inflammation: Stimulating protective innate and/or adaptive immune functions, as well as tissue destruction (21). The results shown in Fig. 1 suggest that proteinases that were upregulated in OSC-20 may play a key role in the cell surface degradation of PD-L1. The results shown in Fig. 2 suggest that several MMPs were upregulated on HNSCC, and in particular, MMP-7 and -13 were upregulated in OSC-20 cells rather than in OSC-19 cells. On the other hand, the OSC-19 cells exhibited a significant upregulation in MMP-9. Indeed, MMP-9 may play important roles in the invasion and metastasis of cancer cells (39-41). However, MMP-9 inhibitors did not exert a significant therapeutic effect in any of these human clinical trials (20). Recent studies have shown a protective role of MMP-9 in carcinogenesis, tumour growth or metastasis, in experiments using MMP-9 KO mice $(42,43)$; however, the detailed mechanisms behind this protective effect remain unclear. The results from the present study demonstrated that PD-L1 shedding in OSC-19 cells was less than that in the OSC-20 cells. We hypotheised that the shedding/cleavage of PD-L1 by MMP-9 was not the main mechanism behind 
the protective effects on invasion and metastasis. The other mechanisms behind the protective effects of MMP-9 warrant further investigation. All of these results suggest that MMP-7 and -13 are strong candidate proteinases for involvement in the cell surface degradation of PD-L1. As shown in Fig. 3, the amount of intact purified PD-L1 was reduced by recombinant MMP-13 and -7 following incubation for $24 \mathrm{~h}$ at $37^{\circ} \mathrm{C}$. There were no digested low-molecular-weight fragments. These results suggest that MMP-13 and MMP-7 have cleavage activity against PD-L1 and PD-L1 digested at multiple regions may be degraded at the PD-1 binding domain. However, the issue of which MMP, MMP-13 or MMP-7, is critical for the cell surface digestion of PD-L1 is remains unclear.

To define which MMP contributes to PD-L1 degradation in HNSCC cells, we used MMP inhibitors with different specificities. As shown in Fig. 4, as the expression of PD-L1 was significantly restored by a specific inhibitor of MMP-13 (CL82198), we hypothesised that MMP-13 was involved in the shedding/cleavage of PD-L1 in the OSC-20 cells. The role of MMP-7 in the shedding/cleavage of PD-L1 may be minor in vitro as the MMP-7 inhibitor, BB94, could not restore cell surface PD-L1 expression. MMP-13 may play a protective role in melanoma cell lung metastasis as the lung metastases of B16BL6 melanoma cells were found to be increased in MMP-13 KO mice after intravenous injection (44). MMP-2, MMP-7, MMP-9 and MMP-14 (MT1-MMP) play important roles in the invasion and metastasis of cancer cells (39-41). Therefore, the targeted deletion of these MMPs may inhibit cancer cell proliferation, invasion and metastasis. Indeed, tumourigenesis and metastasis are decreased in MMP-2 KO (45), MMP-7 KO (46) or MMP-9 $\mathrm{KO}$ mice $(47,48)$. However, contradictory results have also been observed; that is, a protective role of host MMPs in carcinogenesis, tumour growth or metastasis, in experiments using MMP-3 KO (49), MMP-7 KO (48), MMP-9 KO $(42,43)$ and MMP-12 $\mathrm{KO}$ mice (50). In addition, a completely protective role in cancer has been reported in MMP-8 KO mice, which exhibited enhanced carcinogenesis induced by chemical carcinogens (51). Therefore, the data from previous studies suggest that MMP-13 and some MMPs play dual roles in invasion and metastasis, that is, a promoting effect and a protective effect. However, in these previous studies, the detailed mechanisms behind the protective effects on invasion and metastasis were not fully elucidated. The results of this study suggest that the shedding/cleavage of PD-L1 by MMP-13 may be one of the mechanisms behind the protective effects on invasion and metastasis. Further in vivo studies are required to test and confirm this hypothesis.

As shown in Fig. 5, only paclitaxel treatment upregulated MMP-13 expression in HOC313 cells compared with the other tubulin inhibitors tested. No tubulin inhibitor used to treat the OSC-20 and OSC-19 cells increased MMP-13 expression. In a previous study, we determined the in vitro anti-proliferative activities in eribulin-treated OSC-20, OSC-19 and HOC313 cells (52). Of note, the HOC313 cells were highly sensitive to eribulin compared with other cell lines. Previously, another group of researchers demonstrated that the reduced expression levels of 4 tubulins (TUBA1C, TUBA4A, TUBB3 and TUBB6) was significantly associated with eribulin sensitivity and that the expression of one tubulin (TUBB2A) was significantly associated with paclitaxel sensitivity (53). The tubulin inhibitors are broadly classified into microtubule-stabilizing (such as paclitaxel) and microtubule-destabilizing (such as vinblastine and eribulin) drugs (54). SMAD proteins, which are essential mediators of the TGF- $\beta$ signalling pathway, normally bind microtubules (55). SMAD signalling plays an important role in promoting the invasive phenotype of human HNSCC cells by upregulating their MMP-13 expression (56). These data suggest that the expression profiles of tubulins may differ among the OSC-20, OSC-19 and HOC313 cells, and that tubulin inhibitors may stabilise or destabilise the association between SMAD proteins and microtubules in OSC-20, OSC-19 and HOC313 cells, consequently resulting in the differential induction of MMP13 expression. However, we cannot exclude the possibility that paclitaxel may target other molecules to upregulate MMP-13 expression.

Although anti-PD-1 treatment can produce durable responses, it appears to benefit only a subset of patients. If anti-PD-1 therapy alone were not so effective, it would be appropriate to combine this approach with another therapeutic option' to aid readability. However, at present, no candidate drugs for such a combination therapy are known. In this study, we demonstrated that MMP-13 may play a critical role in the shedding/cleavage of PD-L1. In addition, among the anticancer drugs generally used in the treatment of head and neck cancer patients, paclitaxel increased MMP-13 expression in R/M HNSCC cells (HOC313 cells) co-cultured without/with DCs. These results suggested that paclitaxel is a strong candidate for use in combination with anti-PD-1 therapy. Clearly, further in vivo studies are required to test this hypothesis.

\section{Acknowledgements}

We are grateful to the members of the Department of Oral and Maxillofacial Surgery of Kanazawa University for their helpful suggestions and assistance. We would also like to thank Enago (www.enago.jp) for assisting with language editing. This study was supported by Grants-in-Aid for Scientific Research from the Ministry of Education, Science, Sports and Culture, Japan (\#17K11870 to H.K.; \#17K11869 to H.N.; \#15H05042 to S.K.). The funders had no role in the study design, data collection and analysis, decision to publish, or preparation of the manuscript.

\section{References}

1. Topalian SL, Hodi FS, Brahmer JR, Gettinger SN, Smith DC, McDermott DF, Powderly JD, Carvajal RD, Sosman JA, Atkins MB, et al: Safety, activity, and immune correlates of anti-PD-1 antibody in cancer. N Engl J Med 366: 2443-2454, 2012.

2. Brahmer JR, Tykodi SS, Chow LQ, Hwu WJ, Topalian SL, Hwu P, Drake CG, Camacho LH, Kauh J, Odunsi K, et al: Safety and activity of anti-PD-L1 antibody in patients with advanced cancer. N Engl J Med 366: 2455-2465, 2012.

3. Topalian SL, Drake CG and Pardoll DM: Targeting the PD-1/B7-H1(PD-L1) pathway to activate anti-tumor immunity. Curr Opin Immunol 24: 207-212, 2012.

4. Freeman GJ, Long AJ, Iwai Y, Bourque K, Chernova T, Nishimura H, Fitz LJ, Malenkovich N, Okazaki T, Byrne MC, et al: Engagement of the PD-1 immunoinhibitory receptor by a novel B7 family member leads to negative regulation of lymphocyte activation. J Exp Med 192: 1027-1034, 2000.

5. Oliveira-Neto HH, Gleber-Netto FO, de Sousa SF, França CM, Aguiar MC, Silva TA and Batista AC: A comparative study of microvessel density in squamous cell carcinoma of the oral cavity and lip. Oral Surg Oral Med Oral Pathol Oral Radiol 113: 391-398, 2012. 
6. Liang X, Zhou H, Liu X, He Y, Tang Y, Zhu G, Zheng M and Yang J: Effect of local hyperthermia on lymphangiogenic factors VEGF-C and -D in a nude mouse xenograft model of tongue squamous cell carcinoma. Oral Oncol 46: 111-115, 2010.

7. Strome SE, Dong H, Tamura H, Voss SG, Flies DB, Tamada K, Salomao D, Cheville J, Hirano F, Lin W, et al: B7-H1 blockade augments adoptive T-cell immunotherapy for squamous cell carcinoma. Cancer Res 63: 6501-6505, 2003.

8. Lyford-Pike S, Peng S, Young GD, Taube JM, Westra WH, Akpeng B, Bruno TC, Richmon JD, Wang H, Bishop JA, et al: Evidence for a role of the PD-1:PD-L1 pathway in immune resistance of HPV-associated head and neck squamous cell carcinoma. Cancer Res 73: 1733-1741, 2013.

9. Zandberg DP and Strome SE: The role of the PD-L1:PD-1 pathway in squamous cell carcinoma of the head and neck. Oral Oncol 50: 627-632, 2014.

10. Butte MJ, Keir ME, Phamduy TB, Sharpe AH and Freeman GJ Programmed death-1 ligand 1 interacts specifically with the B7-1 costimulatory molecule to inhibit $\mathrm{T}$ cell responses. Immunity 27 111-122, 2007

11. Keir ME, Butte MJ, Freeman GJ and Sharpe AH: PD-1 and its ligands in tolerance and immunity. Annu Rev Immunol 26: 677-704, 2008.

12. Dong H, Strome SE, Salomao DR, Tamura H, Hirano F, Flies DB, Roche PC, Lu J, Zhu G, Tamada K, et al: Tumor-associated B7-H1 promotes T-cell apoptosis: A potential mechanism of immune evasion. Nat Med 8: 793-800, 2002.

13. Tsushima F, Tanaka K, Otsuki N, Youngnak P, Iwai H, Omura K and Azuma M: Predominant expression of B7-H1 and its immunoregulatory roles in oral squamous cell carcinoma. Oral Oncol 42: 268-274, 2006.

14. Iwai Y, Terawaki S and Honjo T: PD-1 blockade inhibits hematogenous spread of poorly immunogenic tumor cells by enhanced recruitment of effector T cells. Int Immunol 17: 133-144, 2005.

15. Hirano F, Kaneko K, Tamura H, Dong H, Wang S, Ichikawa M Rietz C, Flies DB, Lau JS, Zhu G, et al: Blockade of B7-H1 and PD-1 by monoclonal antibodies potentiates cancer therapeutic immunity. Cancer Res 65: 1089-1096, 2005.

16. Hirai M, Kitahara H, Kobayashi Y, Kato K, Bou-Gharios G Nakamura $\mathrm{H}$ and Kawashiri S: Regulation of PD-L1 expression in a high-grade invasive human oral squamous cell carcinoma microenvironment. Int J Oncol 50: 41-48, 2017.

17. Dezutter-Dambuyant C, Durand I, Alberti L, Bendriss-Vermare N, Valladeau-Guilemond J, Duc A, Magron A, Morel AP, Sisirak V, Rodriguez C, et al: A novel regulation of PD-1 ligands on mesenchymal stromal cells through MMP-mediated proteolytic cleavage. Oncoimmunology 5: e1091146, 2015.

18. Egeblad M and Werb Z: New functions for the matrix metalloproteinases in cancer progression. Nat Rev Cancer 2: 161-174, 2002.

19. Overall CM and López-Otín C: Strategies for MMP inhibition in cancer: Innovations for the post-trial era. Nat Rev Cancer 2 : 657-672, 2002

20. Milner JM and Cawston TE: Matrix metalloproteinase knockout studies and the potential use of matrix metalloproteinase inhibitors in the rheumatic diseases. Curr Drug Targets Inflamm Allergy 4: 363-375, 2005.

21. Le NT, Xue M, Castelnoble LA and Jackson CJ: The dual personalities of matrix metalloproteinases in inflammation. Front Biosci 12: 1475-1487, 2007.

22. Yokoi T, Hirata S, Nishimura F, Miyakawa A, Odajima T and Kohama G: Some properties of a newly established human cell line derived from an oral squamous carcinoma. Tumor Res 25 93-91-93, 1990.

23. Yokoi T, Homma $\mathrm{H}$ and Odajima T: Establishment and characterization of OSC-19 cell line in serum and protein free culture. Tumor Res 24: 1-17, 1988.

24. Ishisaki A, Oida S, Momose F, Amagasa T, Rikimaru K, Ichijo H and Sasaki S: Identification and characterization of autocrinemotility-factor-like activity in oral squamous-cell-carcinoma cells. Int J Cancer 59: 783-788, 1994.

25. Arrighi JF, Hauser C, Chapuis B, Zubler RH and Kindler V: Long-term culture of human CD34(+) progenitors with FLT3-ligand, thrombopoietin, and stem cell factor induces extensive amplification of a CD34(-)CD14(-) and a CD34(-) CD14(+) dendritic cell precursor. Blood 93: 2244-2252, 1999.

26. Yang D, Chen Q, Le Y, Wang JM and Oppenheim JJ: Differential regulation of formyl peptide receptor-like 1 expression during the differentiation of monocytes to dendritic cells and macrophages. J Immunol 166: 4092-4098, 2001
27. Livak KJ and Schmittgen TD: Analysis of relative gene expression data using real-time quantitative PCR and the 2(-Delta Delta C(T)) Method. Methods 25: 402-408, 2001.

28. Nakamura H, Fujii Y, Ohuchi E, Yamamoto E and Okada Y: Activation of the precursor of human stromelysin 2 and its interactions with other matrix metalloproteinases. Eur J Biochem 253 67-75, 1998.

29. Nakamura H,Fujii Y, Inoki I, Sugimoto K, Tanzawa K, Matsuki H, Miura R, Yamaguchi Y and Okada Y: Brevican is degraded by matrix metalloproteinases and aggrecanase-1 (ADAMTS4) at different sites. J Biol Chem 275: 38885-38890, 2000

30. Zou W and Chen L: Inhibitory B7-family molecules in the tumour microenvironment. Nat Rev Immunol 8: 467-477, 2008.

31. Wintterle S, Schreiner B, Mitsdoerffer M, Schneider D, Chen L, Meyermann R, Weller M and Wiendl H: Expression of the B7-related molecule B7-H1 by glioma cells: A potential mechanism of immune paralysis. Cancer Res 63: 7462-7467, 2003.

32. Konishi J, Yamazaki K, Azuma M, Kinoshita I, Dosaka-Akita H and Nishimura M: B7-H1 expression on non-small cell lung cancer cells and its relationship with tumor-infiltrating lymphocytes and their PD-1 expression. Clin Cancer Res 10: 5094-5100, 2004.

33. Ohigashi Y, Sho M, Yamada Y, Tsurui Y, Hamada K, Ikeda N, Mizuno T, Yoriki R, Kashizuka H, Yane K, et al: Clinical significance of programmed death-1 ligand-1 and programmed death-1 ligand-2 expression in human esophageal cancer. Clin Cancer Res 11: 2947-2953, 2005

34. Thompson RH and Kwon ED: Significance of B7-H1 overexpression in kidney cancer. Clin Genitourin Cancer 5: 206-211, 2006.

35. Hamanishi J, Mandai M, Iwasaki M, Okazaki T, Tanaka Y, Yamaguchi K, Higuchi T, Yagi H, Takakura K, Minato N, et al: Programmed cell death 1 ligand 1 and tumor-infiltrating CD8 ${ }^{+}$ $\mathrm{T}$ lymphocytes are prognostic factors of human ovarian cancer. Proc Natl Acad Sci USA 104: 3360-3365, 2007.

36. Nomi T, Sho M, Akahori T, Hamada K, Kubo A, Kanehiro H, Nakamura S, Enomoto K, Yagita H, Azuma M, et al: Clinical significance and therapeutic potential of the programmed death-1 ligand/programmed death-1 pathway in human pancreatic cancer. Clin Cancer Res 13: 2151-2157, 2007.

37. Ghebeh H, Tulbah A, Mohammed S, Elkum N, Bin Amer SM, Al-Tweigeri T and Dermime S: Expression of B7-H1 in breast cancer patients is strongly associated with high proliferative Ki-67-expressing tumor cells. Int J Cancer 121: 751-758, 2007.

38. Yao Y, Tao R, Wang X, Wang Y, Mao Y and Zhou LF: B7-H1 is correlated with malignancy-grade gliomas but is not expressed exclusively on tumor stem-like cells. Neuro Oncol 11: 757-766, 2009.

39. Liotta LA and Kohn EC: The microenvironment of the tumourhost interface. Nature 411: 375-379, 2001.

40. Shiomi T and Okada Y: MT1-MMP and MMP-7 in invasion and metastasis of human cancers. Cancer Metastasis Rev 22: $145-152,2003$

41. Deryugina EI and Quigley JP: Matrix metalloproteinases and tumor metastasis. Cancer Metastasis Rev 25: 9-34, 2006.

42. Cornelius LA, Nehring LC, Harding E, Bolanowski M, Welgus HG, Kobayashi DK, Pierce RA and Shapiro SD: Matrix metalloproteinases generate angiostatin: Effects on neovascularization. J Immunol 161: 6845-6852, 1998.

43. Hamano Y, Zeisberg M, Sugimoto H, Lively JC, Maeshima Y, Yang C, Hynes RO, Werb Z, Sudhakar A and Kalluri R: Physiological levels of tumstatin, a fragment of collagen IV alpha3 chain, are generated by MMP-9 proteolysis and suppress angiogenesis via alphaV beta3 integrin. Cancer Cell 3: 589-601, 2003.

44. Fukuda H, Mochizuki S, Abe H, Okano HJ, Hara-Miyauchi C, Okano H, Yamaguchi N, Nakayama M, D'Armiento J and Okada Y: Host-derived MMP-13 exhibits a protective role in lung metastasis of melanoma cells by local endostatin production. Br J Cancer 105: 1615-1624, 2011.

45. Itoh T, Tanioka M, Yoshida H, Yoshioka T, Nishimoto H and Itohara S: Reduced angiogenesis and tumor progression in gelatinase A-deficient mice. Cancer Res 58: 1048-1051, 1998

46. Wilson CL, Heppner KJ, Labosky PA, Hogan BL and Matrisian LM: Intestinal tumorigenesis is suppressed in mice lacking the metalloproteinase matrilysin. Proc Natl Acad Sci USA 94: 1402-1407, 1997.

47. Itoh T, Tanioka M, Matsuda H, Nishimoto H, Yoshioka T, Suzuki R and Uehira M: Experimental metastasis is suppressed in MMP-9-deficient mice. Clin Exp Metastasis 17: 177-181, 1999. 
48. Acuff HB, Carter KJ, Fingleton B, Gorden DL and Matrisian LM: Matrix metalloproteinase-9 from bone marrow-derived cells contributes to survival but not growth of tumor cells in the lung microenvironment. Cancer Res 66: 259-266, 2006.

49. McCawley LJ, Crawford HC, King LE Jr, Mudgett J and Matrisian LM: A protective role for matrix metalloproteinase-3 in squamous cell carcinoma. Cancer Res 64: 6965-6972, 2004.

50. Acuff HB, Sinnamon M, Fingleton B, Boone B, Levy SE, Chen X, Pozzi A, Carbone DP, Schwartz DR, Moin K, et al: Analysis of host- and tumor-derived proteinases using a custom dual species microarray reveals a protective role for stromal matrix metalloproteinase-12 in non-small cell lung cancer. Cancer Res 66: 7968-7975, 2006.

51. Balbín M, Fueyo A, Tester AM, Pendás AM, Pitiot AS, Astudillo A, Overall CM, Shapiro SD and López-Otín C: Loss of collagenase- 2 confers increased skin tumor susceptibility to male mice. Nat Genet 35: 252-257, 2003.

52. Kitahara H, Hirai M, Kato K, Bou-Gharios G, Nakamura H and Kawashiri S: Eribulin sensitizes oral squamous cell carcinoma cells to cetuximab via induction of mesenchymal-to-epithelial transition. Oncol Rep 36: 3139-3144, 2016.
53. Dezső Z, Oestreicher J, Weaver A, Santiago S, Agoulnik S, Chow J, Oda Y and Funahashi Y: Gene expression profiling reveals epithelial mesenchymal transition (EMT) genes can selectively differentiate eribulin sensitive breast cancer cells. PLoS One 9: e106131, 2014.

54. Kavallaris M: Microtubules and resistance to tubulin-binding agents. Nat Rev Cancer 10: 194-204, 2010.

55. Dong C,LiZ, Alvarez R Jr,Feng XH and Goldschmidt-Clermont PJ: Microtubule binding to Smads may regulate TGF-beta activity. Mol Cell 5: 27-34, 2000.

56. Leivonen SK, Ala-Aho R, Koli K, Grénman R, Peltonen J and Kähäri VM: Activation of Smad signaling enhances collagenase-3 (MMP-13) expression and invasion of head and neck squamous carcinoma cells. Oncogene 25: 2588-2600, 2006.

(c) (1) () 9 This work is licensed under a Creative Commons Attribution-NonCommercial-NoDerivatives 4.0 International (CC BY-NC-ND 4.0) License. 